\title{
Virchow 2.0 et la promotion de la santé par les médecins
}

\author{
De l'appel à la mobilisation à une action efficace
}

\author{
Lawrence C. Loh MD MPH FCFP FRCPC Nikhil Rajaram MD CCFP MPH FRCPC
}

L 'intérêt des médecins pour la promotion de la santé a atteint des niveaux sans précédent, et les médias traditionnels et électroniques servent à la mobilisation en faveur d'une diversité de causes ${ }^{1,2}$. D'autres médecins encore ont fait leur entrée en politique $^{3}$, et les principales organisations nationales, comme l'Association médicale canadienne ${ }^{4}$, le Collège des médecins de famille du Canada ${ }^{5}$ et le Collège Royal de médecins et chirurgiens du Canada (CRMCC) ${ }^{6}$, considèrent la promotion de la santé comme une activité importante des médecins, incitant le corps professoral médical à envisager la formation en promotion de la santé comme une composante fondamentale de l'apprentissage.

Un bon nombre des médecins engagés dans la promotion de la santé font souvent référence aux propos de Rudolf Virchow comme étant un cri de ralliement, et rappellent que "la médecine est une science sociale, et que la politique n'est rien d'autre que de la médecine à grande échelle ${ }^{7}$ ». Par ailleurs, après une analyse approfondie de cette partie de la déclaration de Virchow, nous constatons qu'il y a place à interprétation en ce qui a trait à l'engagement des médecins envers la promotion de la santé, que ce soit sur le plan du contenu, de l'approche ou des résultats.

Nous reconnaissons que diverses activités peuvent être considérées comme de la promotion de la santé par les médecins. Le Tableau 1 énonce les compétences clés prévues dans le rôle CanMEDS de promoteur de la santé du CRMCC, et démontre leur large spectre allant de la défense des patients sur le plan individuel jusqu'aux actions qui favorisent des changements systémiques ${ }^{6}$. Quoique la défense des intérêts des patients individuels demeure une pierre angulaire de la pratique qui est relativement simple à mesurer, nous mettons l'accent sur la croissance constante des efforts des médecins pour provoquer des changements d'une envergure systémique. En discutant d'exemples précis d'une telle démarche, nous tentons de proposer des pratiques et des principes qui rendront plus précise et efficace la promotion de la santé par les médecins.

\section{L'efficacité de la promotion}

\section{de la santé par les médecins}

Dans l'exploration de l'efficacité des efforts des médecins à promouvoir la santé, nous proposons d'examiner 2 questions:
- Quelles activités sont considérées comme de la promotion de la santé "de haut niveau" par les médecins?

- Dans quelle mesure ces activités doivent-elles objectivement favoriser de bons résultats en matière de santé?

La définition de haut niveau que propose le CRMCC laisse ouverte la possibilité que "l'efficacité" dans la promotion de la santé par les médecins puisse varier d'un simple plaidoyer en faveur d'une cause jusqu'à la réussite objective d'une incitation à des changements sociétaux de grande envergure. Si nous considérons l'efficacité simplement comme le fait de s'engager, les cas dans lesquels les médecins prennent simplement position sans changement objectif plus large pourraient être considérés efficaces, au même titre que ce que l'on a vu récemment avec les efforts des médecins américains qui préconisaient des mesures de contrôle des armes à feu plus strictes ${ }^{8}$. Toutefois, si nous acceptons le fait que plusieurs médecins s'impliquent souvent dans la promotion de la santé par obligation morale, nous pourrions plutôt définir l'efficacité comme étant mesurée par les résultats observés à la suite des efforts de promotion de la santé au lieu de simplement amplifier une position en particulier.

En mettant l'accent sur les résultats, la prochaine question qui se pose est de savoir dans quelle mesure la promotion de la santé par les médecins devrait faire progresser la santé de manière objective et définitive pour être jugée efficace. Prenons par exemple le débat de longue date entourant le système de santé à 2 vitesses au Canada, où certains médecins argumentent pour et d'autres contre une plus grande implication du secteur privé dans les soins de santé. Des données probantes provenant d'autres pays favorisent largement les défenseurs du régime public, bien que des promoteurs de la médecine à but lucratif soient persuasifs auprès de certains lorsqu'il s'agit de convaincre que leur position améliorera les résultats en matière de santé9.

Étonnamment, la définition du Collège royal pourrait laisser entendre que les 2 parties sont engagées dans une promotion efficace de la santé en soutenant un appel au changement et en parlant au nom d'autres parties concernées en utilisant leur réputation d'experts pour faire avancer leur cause. Nous soutenons toutefois que la promotion de la santé par les médecins n'est véritablement efficace que si elle est utilisée dans le but de faire progresser la santé objectivement. Des données probantes donnent à penser que les médecins 


\section{Tableau 1. Compétences clés pour le rôle de promoteur de la santé dans le référentiel CanMEDS du Collège royal des médecins et chirurgiens du Canada}

Les médecins sont capables de

1. Répondre aux besoins d'un patient en défendant, avec celuici, ses intérêts au sein du milieu clinique et à l'extérieur de celui-ci

2. Répondre aux besoins des collectivités ou des populations servies en collaborant avec celles-ci pour promouvoir, d'une manière socialement responsable, des changements systémiques
1.1 Collaborer avec les patients afin de prendre en compte les déterminants de la santé qui les concernent et qui limitent leur accès aux services de santé ou aux ressources dont ils ont besoin

1.2 Collaborer avec les patients, leur famille et leurs proches aidants pour modifier leurs comportements et adopter de saines habitudes de vie

1.3 Intégrer les principes de prévention de la maladie, et de promotion et de maintien de la santé dans les échanges avec chaque patient

2.1 Collaborer avec des collectivités ou des populations afin de caractériser les déterminants de la santé qui s'y appliquent

2.2 Améliorer la pratique clinique en appliquant un processus d'amélioration continue de la qualité à des activités de prévention de la maladie, et de promotion et de maintien de la santé

2.3 Participer à une initiative d'amélioration de la santé dans la collectivité ou la population qu'ils servent défenseurs du régime public correspondent plus étroitement à la définition d'efficacité.

Tout compte fait, ces éléments portent à croire que la mesure de l'efficacité de la promotion de la santé par les médecins doit se fonder sur la mesure dans laquelle de tels efforts entraînent à la fin de meilleurs résultats sur le plan de la santé, ces derniers étant mesurés indirectement au moyen de paramètres comme la mise en œuvre de programmes et de politiques optimaux en matière de santé.

\section{Attribuer la part des médecins dans la promotion de la santé}

S'il est accepté qu'une promotion efficace de la santé doive être liée à de meilleurs résultats en santé au moyen de changements mesurés dans un contexte, la prochaine étape est de définir clairement l'ampleur de l'implication des médecins et la part qui leur est attribuée.

La définition du Collège royal présente, ici aussi, des difficultés quant à son étendue. Par exemple, l'un des objectifs établis pour les médecins est de promouvoir l'équité en santé, de prévenir la maladie, et de protéger et promouvoir la santé6. Il s'agit, de fait, du mandat de l'ensemble du système de santé publique gouvernemental, ce qui nous porte à nous demander comment un seul médecin promoteur de la santé peut le mieux faire porter ses efforts à la réalisation d'objectifs sur lesquels travaillent déjà des agences de santé publique et des organisations communautaires en entier.

La définition ajoute encore à la confusion en précisant que la promotion de la santé nécessite souvent la mobilisation d'autres intervenants, tout en disant que les médecins pourraient appuyer ou diriger la mobilisation des ressources... à petite ou à grande échelle ${ }^{6}$, ce qui risque de causer de la confusion dans les rôles.
Dans bien des cas, la réalité est que la contribution du médecin n'est que l'une parmi les contributions de nombreux intervenants qui agissent au sein d'interactions complexes entre de nombreux facteurs, comme le moment présent, le contexte, les objectifs gouvernementaux et le travail d'autres parties concernées.

Si nous revenons au débat de la médecine à 2 vitesses au Canada, l'importance de la portée et des attributions pour l'efficacité est bien illustrée dans la biographie publiée d'un récent lauréat d'un prix de l'Association médicale canadienne, qui dit que l'influence singulière des médecins avait probablement empêché l'adoption de modèles de soins à 2 vitesses dans plusieurs provinces au milieu des années $2000^{10}$.

Une telle déclaration pose de nombreux problèmes. Elle ignore le contexte de l'époque, notamment le Plan décennal fédéral pour consolider les soins de santé de $2004^{11}$, qui prévoyait des milliards de dollars pour améliorer l'accès à des services médicaux prioritaires, tout en masquant les contributions de multiples intervenants, comme les infirmières canadiennes, le mouvement syndical et les organisations non médicales et non gouvernementales ${ }^{12}$. Pour d'autres médecins promoteurs de la santé, cette déclaration dresse un portrait d'attentes plutôt irréalistes de ce que leurs propres efforts pourraient accomplir.

À la différence de la défense des intérêts d'un patient à titre individuel, l'efficacité de la promotion de la santé à large échelle par les médecins exige de reconnaître comment le rôle du médecin interagit avec celui d'autres intervenants, comme il est décrit dans l'aspect "partenariat" de la définition du Collège royal. Les médecins promoteurs de la santé sont souvent efficaces parce qu'ils se servent de leur statut privilégié pour se faire le porte-parole de nombreuses mains et voix invisibles sur le terrain. La promotion efficace de 
la santé nécessite par conséquent une introspection approfondie quant au rôle du médecin, tout en assurant que le crédit des réussites dans les contextes changeants soit équitablement accordé entre tous les partenaires impliqués.

\section{Étirer les limites de l'expertise des médecins}

Ayant discuté de l'ampleur et des attributions dans la promotion de la santé, nous nous penchons ensuite sur l'expertise. Dans l'évaluation de l'efficacité, dans quelle mesure les médecins promoteurs de la santé doivent-ils avoir les connaissances et les pouvoirs voulus en ce qui a trait au sujet en cause?

Les médecins sont des experts dans de nombreux domaines en faveur desquels ils plaideront, souvent liés aux soins de santé ou aux sciences biomédicales, mais de nombreux sujets ne font pas partie de leur formation et de leur expérience, ce qui, selon nous, atténue la crédibilité et l'efficacité de leurs efforts. Nous nous rappelons le vieux dicton concernant la "triade" dans laquelle s'embarquent les médecins après leurs études à la faculté de médecine sans formation formelle:l'enseignement, la recherche et la gestion d'une entreprise. Cette situation s'applique cependant à de nombreux autres sujets et activités en dehors de la base de savoir traditionnelle de nombreux médecins.

En voici un exemple: à l'automne de 2017, des changements proposés aux règlements sur l'impôt des corporations privées à contrôle canadien ont mis en opposition 2 camps de médecins, un en faveur et l'autre contre les changements $^{13,14}$. Ce débat a vite dégénéré en une implication typique d'économistes fiscaux et de financiers, et, tandis que certains médecins avaient peut-être cette expertise, les observateurs extérieurs ont probablement vu les 2 camps comme étant respectivement égocentriques et disproportionnés. Autrement dit, si tous les médecins sont des contribuables, tous les médecins ne sont pas nécessairement experts en fiscalité.

Un autre exemple concerne le changement climatique, à l'égard duquel les médecins devraient certainement se prononcer quant à ses effets potentiels sur la santé afin de justifier l'atténuation. Par contre, en l'absence de formation additionnelle, les médecins qui commentent les données probantes et les détails des mesures d'atténuation spécifiques ( $p$. ex. technologies de capture du carbone) pourraient s'engager dans des domaines qui relèvent davantage des experts, comme les ingénieurs et les professionnels du changement climatique.

Une promotion efficace de la santé exige que les médecins plaideurs déterminent si leur expertise sur un sujet leur permet de parler comme un expert plutôt que comme un membre du grand public. Une telle réflexion est conforme aux limites de la portée de la pratique, qui sont fondamentales dans de nombreux autres aspects de la pratique médicale; vous ne verriez habituellement pas un chirurgien général faire de la psychothérapie ni un urgentologue exercer un rôle de leadership en santé publique et en épidémiologie, à moins de suivre une nouvelle formation et d'être évalué.

Une règle d'or pour une promotion de la santé efficace par les médecins serait bien résumée dans la réponse à la question suivante:puis-je parler de ce sujet en tant qu'expert? La réponse permettrait aux médecins de déterminer s'ils sont les mieux placés pour diriger, soutenir une coalition de plaideurs en apportant des points de vue médicaux, ou, dans certains cas, s'abstenir d'exprimer une opinion non éclairée, mais offrir un appui général à la cause.

\section{Rassembler le tout}

En fin de compte, le cri de ralliement communément utilisé en se fondant sur la déclaration de Virchow rate souvent la deuxième moitié de ses réflexions, notamment que la médecine en tant que science sociale, en tant que science des êtres humains, a l'obligation d'attirer l'attention sur les problèmes et de tenter leur solution théorique; le politicien, l'anthropologiste pratique, doit trouver les moyens d'y apporter leur véritable solution ${ }^{7}$. Dans ce contexte plus complet, nous interprétons que cela signifie que l'intention de Virchow n'était pas que les médecins deviennent les seuls défenseurs de tous les maux du monde.

En mentionnant les politiciens, la citation reconnaît que l'exercice ultime dans les décisions au sein des démocraties réside dans l'élection de représentants et la volonté de la population. Elle précise aussi clairement que les médecins ne sont pas nécessairement appelés à diriger et à s'engager à l'égard de chaque problème d'intérêt, mais, plus précisément, à identifier les problèmes et à poser des hypothèses sur les problèmes qui relèvent de la "science de l'être humain".

Nous pouvons donc soutenir que les propos de Virchow décrivent, dans une certaine mesure, l'ampleur d'une promotion de la santé efficace par les médecins, comme nous l'avons proposé dans cet article: un accent mis sur les résultats favorables en santé, et les médecins en tant qu'experts en santé qui éclairent les décisions et les solutions, menant lorsque leur expertise le permet et établissant des partenariats avec des intervenants clés lorsque les éléments à considérer vont au-delà de la seule médecine.

Le $\mathbf{D}^{r}$ Loh est médecin hygiéniste à la Santé publique de Peel à Mississauga (Ontario), et professeur adjoint à l'École Dalla Lana de santé publique à Toronto (Ontario). Le $\mathbf{D}^{r}$ Rajaram est chargé de cours à la Division de médecine occupationnelle du Département de médecine de l'Université de Toronto.

\section{Intérêts concurrents}

Aucun déclaré

Les opinions exprimées dans les commentaires sont celles des auteurs. Leur publication ne signifie pas qu'elles soient sanctionnées par le Collège des médecins de famille du Canada.

\section{Références}

1. Buchman S, Woollard R, Meili R, Goel R. Practising social accountability. From theory to action. Can Fam Physician 2016;62:15-8 (ang), 24-7 (fr). 
2. Bhate TD, Loh LC. Building a generation of physician advocates: the case for including mandatory training in advocacy in Canadian medical school curricula. Acad Med 2015;90(12):1602-6.

3. Collier R. Profile: Dr. Jane Philpott. CMAJ 2016;188(2):100-2.Publ. en ligne du 11 janv. 2016

4. Association médicale canadienne. Health advocacy [site web]. Ottawa, ON: Association médicale canadienne. Accessible à: https://www.cma.ca/health-advocacy. Réf. du 8 sept. 2019.

5. Collège des médecins de famille du Canada. Four principles of family medicine. Mississauga, ON: Collège des médecins de famille du Canada. Accessible à : https:// www.cfpc.ca/Principles/. Réf. du 8 sept. 2019.

6. Sherbino J, Bonnycastle D, Côté B, Flynn L, Hunter A, Ince-Cushman D, et coll. Health advocate. Dans: Frank JR, Snell L, Sherbino J, rédacteurs. CanMEDS 2015 physician competency framework. Ottawa, ON: Collège royal des médecins et chirurgiens du Canada; 2015.

7. Virchow RC. Collected essays on public health and epidemiology. Vol 1. Rather LJ, rédacteur. Boston, MA: Science History Publications; 1985.

8. Pane LM. It's a Twitter war: doctors clash with NRA over gun deaths. Associated Press du 21 nov. 2018. Accessible à : https://www.apnews.com/ef9238dd2b 75447d8fc1c0b3144d508f. Réf. du 8 sept. 2019.

9. Mertl S. BC refutes Charter challenge of Medicare. CMAJ 2016;188(15):E369-70. Publ. en ligne du 19 sept. 2016.

10. Association médicale canadienne. F.N.G. Starr Award-2019 recipient. Ottawa, ON: Association médicale canadienne; 2019. Accessible à: https://www.cma.ca/f-n-gstarr-award-2019-recipient. Réf. du 8 sept. 2019.
11. A 10-year plan to strengthen health care. Ottawa, ON: gouvernement of Canada; 2004. Accessible à : https://www.canada.ca/en/health-canada/services/ health-care-system/health-care-system-delivery/federal-provincial-territorialcollaboration/first-ministers-meeting-year-plan-2004/10-year-plan-strengthenhealth-care.html. Réf. du 30 oct. 2020.

12. Musée canadien de l'histoire. Making Medicare: the history of health care in Canada 1914-2007. Gatineau, QC: Musée canadien de l'histoire; 2010. Accessible à : https:// www.historymuseum.ca/cmc/exhibitions/hist/medicare/medic-8h21e.html. Réf. du 8 sept. 2019

13. Ontario Medical Association. Proposed federal tax changes. Toronto, ON: Ontario Medical Association; 2019

14. An open letter to Minister Morneau. From physicians and medical students with a shared perspective on the proposed tax changes [site web]. Accessible à: https:// docsandtaxes.wordpress.com/. Réf. du 8 sept. 2019.

Cet article a fait l'objet d'une révision par des pairs. Can Fam Physician 2020;66:887-90. DOI: 10.46747/cfp.6612887

This article is also in English on page 884. 